\title{
Produção e qualidade de frutos de tomateiros portadores de alelos mutantes de amadurecimento e coloração
}

\author{
Valter Carvalho de Andrade Júnior ${ }^{(1)}$, Wilson Roberto Maluf(2), Marcos Ventura Faria(3), \\ Flávio Rodrigo Gandolfi Benites ${ }^{(2)}$ e Alcides Militão dos Santos Júnior ${ }^{(4)}$
}

\begin{abstract}
(1)Faculdades Federais Integradas de Diamantina, Fac. de Ciências Agrárias, Dep. de Agronomia, Rua da Glória, no 187, Centro, CEP 39100-000 Diamantina, MG. E-mail: valterjr@fafeid.edu.br (2)Universidade Federal de Lavras, Dep. de Agricultura e Biologia, Caixa Postal 37, CEP 37200-000 Lavras, MG. E-mail: wrmaluf@ufla.br, frg.benites@yahoo.com.br(3)Universidade do Centro Oeste, Dep. de Agronomia, Rua Presidente Zacarias, no 875, Caixa Postal 3010, CEP 85015-430 Guarapuava, PR. E-mail: mfaria@unicentro.br (4)Escola Agrotécnica Federal de Vitória de Santo Antão, Propriedade Terra Preta - Zona Rural, Caixa Postal 031, CEP 55600-000 Recife, PE. E-mail: alcidesmilitaojr@vk.com.br
\end{abstract}

Resumo - Sete híbridos de tomateiros quase-isogênicos, à exceção dos locos nor ${ }^{A} /$ nor, $r i n, o g^{c}$ e $h p$, com as linhagens parentais FloraDade e Mospomorist, e dois híbridos comerciais heterozigotos no loco rin (Carmen $\mathrm{F}_{1}$ e Chronos $\mathrm{F}_{1}$ ) foram avaliados quanto às características de produção e qualidade de frutos e quanto aos possíveis efeitos do background genotípico empregado nas mesmas características. Foi utilizado o delineamento em blocos casualizados, com quatro repetições e dez plantas por parcela. Os genótipos nor $^{+} /$nor e in $^{+} /$rin não $^{2}$ afetaram as características de produção. O genótipo nor ${ }^{+} / n o r^{A}$ atuou diminuindo a massa média por fruto. Os genótipos nor ${ }^{+} /$nor $^{A}$, nor ${ }^{+} /$nor e $\mathrm{rin}^{+} /$rin, isoladamente, atrasaram a perda de firmeza e a chegada da coloração vermelha nos frutos. O tamanho relativo da cicatriz peduncular não foi afetado significativamente por esses genótipos. A combinação $\mathrm{og}^{c+} / \mathrm{og}^{c} h p^{+} / \mathrm{hp}$ proporcionou maior produção total e maior massa média por fruto no híbrido nor $^{+} /$nor $^{A}$. A firmeza e a coloração dos frutos nor $/$ nor $^{A}$ não foram afetadas pela combinação $o g^{c+} / \operatorname{og}^{c}$ $h p^{+} / \mathrm{hp}$. O genótipo $n \mathrm{rr}^{+} / \mathrm{nor}^{A}$ reduziu a produção precoce de frutos $\mathrm{og}^{c+} / \mathrm{og}^{c} \mathrm{hp} \mathrm{p}^{+} / \mathrm{hp}$ e aumentou a meia-vida da firmeza desses frutos. O background genotípico e a interação background x mutante de amadurecimento devem ser considerados na produção de híbridos $\mathrm{F}_{1}$ de tomateiro.

Termos para indexação: Lycopersicon esculentum, híbridos, conservação pós-colheita, firmeza, melhoramento de plantas.

\section{Yield and fruit quality of tomato hybrids heterozygous for ripening and color mutant alleles}

\begin{abstract}
Seven nearly isogenic tomato hybrids, except for their genotypic constitutions in loci nor ${ }^{A} /$ nor, rin, $o g^{c}$ and $h p$, were tested for fruit yield and quality traits along the parental background lines FloraDade and Mosporist and two commercial $\mathrm{rin}^{+} /$rin tomato hybrids (Carmen $\mathrm{F}_{1}$ and Chronos $\mathrm{F}_{1}$ ). The role of the different genotypic backgrounds on these fruit traits was also studied. The genotypes were tested using a randomized complete block design with four replicates and ten plants per plot. The genotypic constitutions nor $^{+} /$nor $\mathrm{or} \mathrm{rin}^{+} /$ rin had no effect on yield related traits. The genotype nor $^{+} /$nor $^{A}$ decreased fruit mass mean. Genotypes nor ${ }^{+} /$ nor $^{\mathrm{A}}$, nor ${ }^{+} /$nor and $\mathrm{rin}^{+} /$rin delayed the loss of fruit firmness and the development of color. The relative size of the peduncular scar was not affected by these genotypes. The constitution $\mathrm{og}^{c+} / \mathrm{og}^{c} \mathrm{hp} / \mathrm{hp}$ contributed for higher yield and higher fruit mass in the nor ${ }^{+} /$nor $^{A}$ hybrid. Fruit firmness and external color in $\operatorname{og}^{c+} / \mathrm{og}^{c} h p^{+} / \mathrm{hp}$ remained unaffected by nor $/ \mathrm{nor}^{A}$. In $\mathrm{og}^{c^{+}} / \mathrm{og}^{c} \mathrm{hp^{+ }} / \mathrm{hp}$ fruit, early yield was reduced and shelf life was improved by nor $^{+} /$nor $^{A}$. Genotypic backgrounds and the interaction background x ripening mutant genes must be taken into consideration when developing new tomato long-shelf life hybrids.
\end{abstract}

Index terms: Lycopersicon esculentum, hybrids, post-harvest shelf life, firmness, plant breeding.

\section{Introdução}

A utilização de mutantes de amadurecimento pelos melhoristas visa a aumentar a firmeza e a conservação pós-colheita dos frutos do tomateiro. Os alelos mutantes que atuam de forma marcante sobre a conservação póscolheita dos frutos são: alcobaça (nor $\left.{ }^{A}\right)$, ripening inhibitor (rin) e non ripening (nor). Há também os alelos high pigment ( $h p$ ) e old gold crimson $\left(\mathrm{og}^{c}\right)$, que aumentam a produção de carotenóides nos frutos, e podem ser usados em um mesmo genótipo, aliados aos alelos mutantes de amadurecimento. Araujo et al. (2002) concluíram que o alelo alcobaça em homozigose (alc/alc) prolonga a vida pós-colheita, pela redução da 
perda de peso e aumento da firmeza, reduz os teores de licopeno e beta-caroteno e aumenta a relação brix/acidez, porém sua coloração externa limita sua utilização comercial. Em heterozigose $\left(\right.$ alc $^{+} /$alc $)$, o alelo alcobaça não prejudicou a coloração dos frutos, contudo, reduziu a perda de peso e aumentou a firmeza, principalmente em associação com os genótipos $h p / h p$ e $h p^{+} / h p$ ou com $o g^{c} / \mathrm{og}^{c}$. Segundo os autores as interações entre $o g^{c}$ e $h p$ com o alelo alc heterozigoto (alc $/$ alc) se revelaram promissoras em manter ou aumentar o número de frutos comerciáveis.

Leal \& Mizubuti (1975) atribuíram a menor perda de peso de frutos alc $^{+} / \mathrm{alc}^{+}$e alc $\mathrm{C}^{+} / \mathrm{alc}$ ao menor tamanho de cicatriz peduncular. Tal perda, baseada na perda de água dos frutos, decorre da alta taxa de respiração do tomate verificada através da cicatriz peduncular. A perda de água do tomate, além da ação das enzimas poligalacturonase e pectinametilesterase (Resende et al., 1997), promove amaciamento e murchamento dos frutos, reduzindo sua vida pós-colheita. Assim, efeitos pleiotrópicos do alelo alc, que reduzem o tamanho da cicatriz peduncular (Freitas et al., 1999) e a perda de peso dos frutos (Freitas et al., 1999; Araujo et al., 2002), explicam em parte a maior firmeza dos tomates alc $^{+} / a l c$, comparada àquelas dos frutos normais $\left(a c^{+} / a l c^{+}\right)$, cujo tamanho de cicatriz peduncular foi maior. Freitas et al. (1998), estudando os parentais (alc/alc), $\left(a c^{+} / a l c^{+}\right)$e o híbrido $\mathrm{F}_{1}\left(\right.$ alc $^{+} /$alc), verificaram uma ação gênica de dominância parcial ou de baixo grau, do alelo alc, que reduz o tamanho de cicatriz peduncular dos frutos de tomateiro. A hipótese de um efeito de background genético, que resultasse em um menor tamanho de cicatriz peduncular, poderia contribuir para a redução de perda de peso dos frutos híbridos, favorecendo uma vida de prateleira prolongada.

Benites (2003) demonstrou que alc e nor são alelos semelhantes ou idênticos do mesmo loco, e que alc deveria ser denominado nor ${ }^{A}$. O autor verificou que o uso do genótipo $\mathrm{rin}^{+} /$rin ou das duplas combinações alc/alc $\mathrm{rin}^{+} /$rin e nor $/$nor $\mathrm{rin}^{+} /$rin, em programas de melhoramento para produção de híbridos de tomate longa vida, torna-se viável, apesar do ligeiro atraso na evolução da coloração interna dos frutos, embora a magnitude deste atraso não chegasse a impedir seu emprego comercial.

Outro fator que deve ser considerado na produção de híbridos $\mathrm{F}_{1}$ de tomateiro do tipo longa vida é o background genotípico, pois quando os frutos atingem o estádio final de amadurecimento podem ser firmes ou macios, dependendo do background genotípico ou da presença ou ausência de genes que afetam especificamente a firmeza (Kopeliovitch et al., 1979; Freitas et al., 1998; Freitas et al., 1999; Andrade-Júnior et al., 2001; Araujo et al., 2002; Santos-Júnior, 2002; Benites, 2003; Faria et al., 2003). Embora seja relatada a importância do background genotípico na maior conservação póscolheita dos frutos, nenhum trabalho separou os efeitos dos mutantes de amadurecimento do efeito do background sobre a conservação pós-colheita de frutos de tomateiro.

O objetivo deste trabalho foi avaliar a produção e a qualidade de frutos híbridos de tomateiros portadores de alelos mutantes de amadurecimento e de coloração, e estimar os possíveis efeitos do background genotípico empregado nas mesmas características.

\section{Material e Métodos}

Utilizou-se para o mutante alcobaça a notação nor ${ }^{A}$ proposta por Lobo (1981), uma vez que sua relação de alelismo com nor, ao contrário do que relatou Mutschler (1984), foi confirmada por Benites (2003).

Sete linhagens quase-isogênicas de tomateiro, obtidas a partir de retrocruzamentos, com background FloraDade [FloraDade $\left(\mathrm{nor}^{+} / \mathrm{nor}^{+} \mathrm{rin}^{+} / \mathrm{rin}^{+} \mathrm{og}^{\mathrm{C}^{+}} / \mathrm{og}^{\mathrm{C}^{+}}\right.$ $\mathrm{hp}^{+} / \mathrm{hp}^{+}$), TOM-596 (nor $/$nor $^{+} \mathrm{rin}^{+} / \mathrm{rin}^{+} \mathrm{og}^{\mathrm{C}} / \mathrm{og}^{\mathrm{C}} \mathrm{hp}^{+} / \mathrm{hp^{+ }}$ ), TOM-588 (nor $/$ nor $\left.^{+} \mathrm{rin}^{+} / \mathrm{rin}^{+} \mathrm{og}^{\mathrm{c}} / \mathrm{og}^{\mathrm{c}} \mathrm{hp} / \mathrm{hp}\right)$, TOM-559 (nor ${ }^{A} /$ nor $^{A}$ rin $^{+} /$rin $^{+}$og $^{c^{+}} /$og $^{c^{+}} h^{+} / \mathrm{hp}^{+}$), TOM-589 (nor $/$ nor $^{A}$ rin $^{+} /$rin $^{+}$og $^{c} /$ og $^{c}$ hp/hp), TOM-613 (nor/nor rin ${ }^{+} / \mathrm{rin}^{+} \mathrm{og}^{\mathrm{c}^{+}} / \mathrm{og}^{\mathrm{c}} \mathrm{hp}^{+} / \mathrm{hp}^{+}$, TOM-614 (nor $/$ nor $^{+}$rin/rin og $\left.{ }^{c+} / \mathrm{og}^{c^{+}} \mathrm{hp}^{+} / \mathrm{hp}^{+}\right)$] foram utilizadas como genitores femininos em combinações com a linhagem Mospomorist (fonte de pólen), originando sete híbridos experimentais quase-isogênicos, com constituições genotípicas: $\mathrm{F}_{1}$ (FloraDade $\mathrm{x}$ Mospomorist $)=$ nor $^{+} /$nor $^{+} \mathrm{rin}^{+} / \mathrm{rin}^{+} \mathrm{og}^{\mathrm{c}^{+}} / \mathrm{og}^{\mathrm{c}^{+}} \mathrm{hp}^{+} / \mathrm{hp}^{+}$ $=$ normal; $\mathrm{F}_{1}(\mathrm{TOM}-596 \mathrm{x}$ Mospomorist $)=$ nor $^{+} /$nor $^{+}$ $\mathrm{rin}^{+} / \mathrm{rin}^{+} \mathrm{og}^{c+} / \mathrm{og}^{c} \mathrm{hp}^{+} / \mathrm{hp}^{+}=$heterozigoto $\mathrm{og}^{c}$; $\mathrm{F}_{1}(\mathrm{TOM}-588 \times$ Mospomorist $)=$ nor $^{+} /$nor $^{+} \mathrm{rin}^{+} / \mathrm{rin}^{+}$ $\mathrm{og}^{c+} / \mathrm{og}^{c} h p^{+} / \mathrm{hp}=$ heterozigoto $o g^{c} \& h p ; \mathrm{F}_{1}$ (TOM $559 \mathrm{x}$ Mospomorist $)=$ nor $^{+} /$nor $^{A} \mathrm{rin}^{+} / \mathrm{rin}^{+} \mathrm{og}^{\mathrm{c}^{+}} / \mathrm{og}^{\mathrm{C}^{+}} \mathrm{hp}^{+} / \mathrm{hp}^{+}$ $=$ heterozigoto nor ${ }^{A} ; \mathrm{F}_{1}$ (TOM-589 $\mathrm{x}$ Mospomorist $)=$ nor $^{+} /$nor $^{A}$ rin $^{+} /$rin $^{+}$og $^{c+} /$ og $^{c} \mathrm{hp}^{+} / \mathrm{hp}=$ heterozigoto nor $^{A}$ $\& o g^{c} \& h p ; \mathrm{F}_{1}(\mathrm{TOM}-613 \times$ Mospomorist $)=$ nor $^{+} /$nor $\mathrm{rin}^{+} / \mathrm{rin}^{+} \mathrm{og}^{\mathrm{c}^{+}} / \mathrm{og}^{\mathrm{c}^{+}} \mathrm{hp}^{+} / \mathrm{hp}^{+}=$heterozigoto nor; $\mathrm{F}_{1}$ $($ TOM-614 x Mospomorist $)=$ nor $^{+} /$nor $^{+} \mathrm{rin}^{+} / \mathrm{rin} \mathrm{og}^{\mathrm{c}^{+}} / \mathrm{og}^{\mathrm{c}^{+}}$ $h p^{+} / h p^{+}=$heterozigoto rin. FloraDade é uma cultivar de crescimento determinado, "jointless" $\left(\mathrm{j}_{2} / \mathrm{j}_{2}\right)$, de frutos 
multiloculares, criada na Universidade da Flórida, USA. Mospomorist é uma linhagem do tipo Moneymaker, obtida pelo INRA, Avignon, França, de amadurecimento normal, com resistência múltipla a doenças e de crescimento indeterminado.

Foram avaliados 11 genótipos, sendo sete os híbridos experimentais de background FloraDade x Mospomorist anteriormente descritos, dois híbridos comerciais longa vida heterozigotos no loco rin (Carmen $\mathrm{F}_{1}$ e Chronos $\mathrm{F}_{1}$ ) e as linhagens FloraDade e Mospomorist. O delineamento experimental utilizado foi em blocos casualizados completos, contendo 11 tratamentos com quatro repetições e dez plantas por parcela, perfazendo um total de 440 plantas. Cada parcela constituiu de uma fileira única de $5,0 \mathrm{~m}$ de comprimento por $0,70 \mathrm{~m}$ de largura $\left(3,5 \mathrm{~m}^{2}\right)$.

O trabalho foi desenvolvido na Universidade Federal de Lavras (Ufla) ao longo dos anos 2000 e 2001. As sementes correspondentes aos 11 genótipos foram semeadas em caixas de plástico contendo substrato Plantimax ${ }^{\circledR}$ à base de vermiculita e casca de Pinus. Posteriormente, efetuou-se a repicagem das plântulas para bandejas de isopor de 128 células que continham substrato Platimax ${ }^{\circledR}+$ casca de arroz carbonizada na proporção de 1/1 e 800 g de adubo na formulação 4:14:8 para cada $80 \mathrm{~L}$ desta mistura. $\mathrm{O}$ transplantio das mudas foi realizado aproximadamente 30-40 dias após a semeadura. As mudas foram transplantadas para canteiros em fila dupla com espaçamento de $70 \mathrm{~cm}$ entre linhas e $50 \mathrm{~cm}$ entre plantas. O plantio foi feito em sistema tutorado e a irrigação, por gotejamento. As plantas foram estaqueadas com bambu e conduzidas com duas hastes principais, sendo desbrotadas semanalmente com poda no ápice, uma folha acima do quinto rácimo floral. Os demais tratos culturais foram realizados de acordo com as recomendações para a cultura do tomateiro em ambiente protegido.

A colheita foi iniciada quando os primeiros frutos atingiram mudanças perceptíveis na coloração (estádio breaker) e prosseguiu semanalmente, sendo avaliadas as características de produção e qualidade dos frutos.

A produção total de frutos foi obtida pelo somatório das massas de todos os frutos de cada parcela durante as sucessivas colheitas. Já a produção precoce de frutos foi obtida somando-se as massas dos frutos de cada parcela nos primeiros vinte dias de colheita. Os resultados de produção total e produção precoce de frutos foram expressos em $t$ ha $^{-1}$.
A massa média por fruto foi determinada dividindose a massa total dos frutos de cada parcela, pelo número total de frutos da respectiva parcela, durante as sucessivas colheitas. Os resultados foram expressos em g fruto $^{-1}$. Nas avaliações de qualidade de frutos foram amostrados dez frutos por parcela colhidos no estádio breaker de amadurecimento.

O número médio de dias da antese ao estádio breaker foi avaliado no período do florescimento das plantas, e, a cada dois dias, foram marcadas, com lã colorida, as flores abertas naquela data (a cada data, uma cor correspondente), totalizando no mínimo vinte flores por parcela. Os frutos foram colhidos no estádio breaker, sendo registrados os dias decorridos desde a antese correspondente. Para efeito de análise estatística, a média da parcela correspondeu à média dos dez frutos amostrados.

O tamanho relativo da cicatriz peduncular foi obtido por meio da determinação das medidas do maior diâmetro da cicatriz peduncular e do diâmetro de cada fruto amostrado utilizando-se um paquímetro. A razão entre essas duas medidas constituiu o diâmetro da cicatriz peduncular relativamente ao diâmetro dos frutos, sendo os resultados expressos em porcentagem.

Em relação à caraceterística firmeza, os frutos foram armazenados em câmara fria com temperatura controlada $\left(15^{\circ} \mathrm{C}\right)$ e umidade relativa de $60 \%$, em prateleiras, onde permaneceram durante todo o período das avaliações. Os frutos foram avaliados quanto à firmeza pela técnica de aplanação descrita por Calbo \& Nery (1995). As medidas de firmeza foram expressas em $\mathrm{N} \mathrm{m}^{-2}$.

A coloração dos frutos foi avaliada por observação visual. Os dez frutos amostrados de cada parcela receberam notas individuais, a cada dois dias, quanto ao grau de coloração externa, dentro de uma escala de 1 a 5 , conforme a seguir: 1: frutos no breaker stage, ou seja, com poucas listras ou manchas de coloração vermelha; 2: frutos com $20 \%$ a $40 \%$ da superfície de área com coloração vermelha; 3 : frutos com $40 \%$ a $60 \%$ da superfície de área com coloração vermelha; 4: frutos com $60 \%$ a $80 \%$ da superfície de área com coloração vermelha; 5: frutos com mais de $80 \%$ da área de superfície com coloração vermelha.

Como os híbridos experimentais avaliados eram quase-isogênicos, foram calculados contrastes nãoortogonais de interesse entre os pares de híbridos a fim de avaliar os efeitos dos genes nor ${ }^{A} /$ nor, rin, e $o g^{c}$, em heterozigose, isoladamente, bem como os efeitos das combinações de vários destes locos em heterozigose, 
simultaneamente $\left(o g^{c}+h p ; n o r^{A}+o g^{c}+h p\right)$. Os contrastes calculados entre o híbrido experimental $\mathrm{F}_{1}$ (TOM-614 x FloraDade) e os híbridos comerciais Carmen $\mathrm{F}_{1}$ e Chronos $\mathrm{F}_{1}$, todos heterozigotos para o loco rin $\left(\mathrm{rin}^{+} / \mathrm{rin}\right)$, refletem o efeito do background genotípico sobre as características avaliadas, livre dos possíveis efeitos do alelo mutante rin.

A evolução da coloração dos frutos foi medida anotando-se, para cada parcela, o número de dias para atingir as notas 4 e 5 de coloração. A meia-vida de firmeza (T) foi obtida por meio da regressão dos dados de firmeza (A), de cada parcela, no número de dias decorridos (X), pelo modelo estatístico do decaimento exponencial: $\mathrm{A}=\mathrm{A}_{0}(1 / 2)^{\mathrm{X} / \mathrm{T}}$, em que $\mathrm{A}_{0}$ é a firmeza inicial $\left(\mathrm{N} \mathrm{m}^{-2}\right)$ dos frutos no estádio breaker (equivale ao dia 0); X é o número de dias decorridos após colheita no estádio breaker; T é a meia-vida da firmeza (em dias); e A é a firmeza $\left(\mathrm{N} \mathrm{m}^{-2}\right)$ após decorridos $\mathrm{X}$ dias. As regressões foram calculadas com o recurso do pacote estatístico SAS, utilizando-se para o modelo de decaimento exponencial, a transformação logarítmica seguida de regressão linear. Os valores obtidos para a firmeza de frutos, ao longo do tempo decorrido após a colheita, foram ajustados ao modelo de decaimento exponencial indicado. Com base na equação de regressão ajustada, foram estimadas para cada parcela: a firmeza inicial do fruto no estádio breaker $\left(\mathrm{A}_{0}\right)$; a meia-vida da firmeza (T); e o número médio de dias para os frutos atingirem a firmeza $3,0 \times 10^{4} \mathrm{~N} \mathrm{~m}^{-2}$.

\section{Resultados e Discussão}

Foram observados valores heteróticos altos para as características de produção total (62,85\%) e produção precoce $(52,13 \%)$ (Tabela 1$)$. Faria et al. (2003) registraram valores de heterose de $34,37 \%$ e $7,45 \%$, respectivamente, para produção total e precoce.

Pela análise dos contrastes (Tabela 1), constatou-se que os genótipos nor ${ }^{+} /$nor e $\mathrm{rin}^{+} /$rin não afetaram a produção total, produção precoce e a massa média por fruto. Já o genótipo or $^{+} /$nor $^{A}$ atuou para diminuir a massa média por fruto. Contudo, na combinação nor $^{+} /$nor $^{A}$ $\mathrm{og}^{c+} / \mathrm{og}^{c} h \mathrm{p}^{+} / \mathrm{hp}$, o genótipo nor $^{+} /$nor $^{A}$ não afetou negativamente essas características, indicando que, juntamente com os genótipos $\mathrm{og}^{c+} / \mathrm{og}^{c}$ e $h p^{+} / h p$, na mesma combinação, o mutante nor ${ }^{A}$, em heterozigose, pode ser usado sem afetar negativamente estas características. Araujo et al. (2002) relataram que o alelo nor ${ }^{A}$ em homozigose e também em heterozigose apresentou efeito negativo sobre a massa média por fruto. Já Santos-Júnior (2002) e Faria et al. (2003) não verificaram influência do genótipo $n o r^{+} /$nor $^{A}$ sobre a massa média por fruto.

Embora não significativa, a colheita precoce de frutos sofreu alguma influência negativa dos alelos nor ${ }^{A}$, rin e nor, em heterozigose (Tabela 1). Essa menor produção precoce está relacionada, em parte, à contribuição dos locos nor $^{+} /$nor $^{A}$, nor ${ }^{+} /$nor e $\mathrm{rin}^{+} /$rin, no sentido de retardar o início do estádio breaker e prolongar a permanência dos frutos na planta. O genótipo nor ${ }^{+} /$nor $^{A}$ diminuiu significativamente a produção precoce de frutos $o g^{c^{+}} / \operatorname{og}^{c} h p^{+} / h p$, o que pode ser comprovado pelo maior período de permanência dos frutos nas plantas $\mathrm{og}^{c^{+}} / \mathrm{og}^{c} \mathrm{hp} \mathrm{p}^{+} / \mathrm{hp}$, proporcionado pelo genótipo nor $^{+} /$nor $^{A}$. O genótipo nor $^{+} /$nor $^{A}$ e a combinação nor $^{+} /$nor $^{A}$ og $^{c^{+}} / \log ^{c}$ $h p^{+} / h p$ aumentaram significativamente o período de permanência dos frutos nas plantas quando comparados com o genótipo normal.

A combinação $\operatorname{og}^{c+} / \operatorname{og}^{c} h p^{+} / h p$ contribuiu para aumentar a produção total e a massa média por fruto do híbrido nor $^{+} /$nor $^{A}$. Resultados semelhantes foram obtidos por Araujo et al. (2002) quanto à produção total de frutos. No entanto, Faria et al. (2003) não verificaram efeito significativo da combinação $o g^{c^{+}} / \mathrm{og}^{c} \mathrm{hp} \mathrm{p}^{+} / \mathrm{hp}$ sobre a produção de frutos e sobre a massa média por fruto em híbrido nor $^{+} /$nor $^{A}$ de background FloraDade x Mospomorist.

O genótipo $\mathrm{rin}^{+} /$rin apresentou maior produção total em relação ao genótipo $n o r^{+} /$nor $^{A}$ e os genótipos nor $^{+} /$nor e $\mathrm{rin}^{+} /$rin apresentaram maior massa média por fruto quando comparados com o genótipo nor $^{+} /$nor $^{A}$. Já os genótipos nor ${ }^{+}$nor e rin $^{+} /$rin não apresentaram diferenças entre si quanto às características de produção de frutos.

O tamanho relativo da cicatriz peduncular não foi afetado significativamente pelos genótipos nor $^{+} /$nor $^{A}$, nor $^{+} /$nor e rin $^{+} /$rin (Tabela 1). Resultados semelhantes foram obtidos por Faria et al. (2003) em relação ao genótio nor $^{+} /$nor $^{A}$. Freitas et al. (1998) e Freitas et al. (1999) verificaram que o genótipo nor $^{+} /$nor $^{A}$ atuou na redução do tamanho de cicatriz peduncular de frutos. Araujo et al. (2002) relacionaram a menor perda de água em pós-colheita e a maior vida de prateleira dos frutos nor $^{A}$ heterozigotos à redução do tamanho da cicatriz peduncular.

Os genótipos nor $^{+} /$nor $^{A}$, nor ${ }^{+} /$nor e rin $^{+} /$rin promoveram maior meia-vida da firmeza dos frutos no background utilizado. Os genótipos nor ${ }^{+} /$nor $^{A}$ e rin $^{+} /$rin 
apresentaram efeitos semelhantes no prolongamento da meia-vida da firmeza dos frutos, em 2,9 dias, em relação ao genótipo normal (Tabela 2). Já o genótipo nor ${ }^{+} /$nor proporcionou aumento de 1,8 dias na meia-vida da firmeza dos frutos. Santos-Júnior (2002), trabalhando com background distinto, verificou que o alelo nor $^{A}$ em heterozigose não apresentou efeito favorável sobre a firmeza dos frutos.

Não foram observadas diferenças significativas entre os genótipos nor ${ }^{+} /$nor $^{A}$, nor ${ }^{+} /$nor e rin $^{+} /$rin quanto à maior conservação pós-colheita dos frutos. No trabalho realizado por Santos-Júnior (2002), o genótipo $\mathrm{rin}^{+} /$rin mostrou-se mais promissor quando comparado com o genótipo nor ${ }^{+} /$nor, proporcionando maior meia-vida da firmeza.

A combinação $n r^{+} /$nor $^{A} o^{c+} / o g^{c} h p^{+} / h p$ foi eficiente em reduzir a perda de firmeza dos frutos, assim como a meia-vida da firmeza de frutos $\mathrm{og}^{\mathrm{c}} / \mathrm{og}^{\mathrm{c}} \mathrm{hp} \mathrm{p}^{+} / \mathrm{hp}$ foi aumentada pelo genótipo nor $^{+} /$nor $^{A}$.

Os genótipos nor $^{+} /$nor $^{A}$ e $n o r^{+} /$nor apresentaram efeitos semelhantes sobre a coloração dos frutos, com atuação no atraso da chegada da coloração vermelha dos frutos em relação ao genótipo normal. $\mathrm{O}$ alelo rin, em heterozigose, isoladamente retardou a chegada da pigmentação vermelha intensa dos frutos nos estágios mais avançados de amadurecimento. Os frutos do genótipo $\mathrm{rin}^{+} /$rin demoraram mais para atingir a coloração vermelha intensa quando comparados com os frutos dos genótipos nor ${ }^{+} /$nor $^{A}$ e nor $^{+} /$nor. Os genótipos nor $^{+} /$nor e nor $^{+} /$nor $^{A}$ não apresentaram diferenças entre si quanto à coloração externa dos frutos, podendo ser um indicativo de que os mutantes nor ${ }^{A}$ e nor são alelos, mas não idênticos.

Tabela 1. Valores médios relativos à produção total (PT), produção precoce (PP), massa média por fruto (MMF), número médio de dias da antese ao estádio breaker, tamanho relativo da cicatriz peduncular (TRCP) e contrastes não-ortogonais de interesse entre 11 genótipos de tomateiro ${ }^{(1)}$.

\begin{tabular}{|c|c|c|c|c|c|c|}
\hline Tratamento & Genótipo & $\begin{array}{c}\text { PT } \\
\left(\mathrm{t} \mathrm{ha}^{-1}\right)\end{array}$ & $\begin{array}{c}\text { PP } \\
\left(\mathrm{t} \mathrm{ha}^{-1}\right)\end{array}$ & $\begin{array}{c}\text { MMF } \\
\left(\mathrm{g} \mathrm{fruto}^{-1}\right)\end{array}$ & $\begin{array}{c}\text { Antese ao estádio } \\
\text { breaker (dias) }\end{array}$ & $\begin{array}{c}\text { TRCP } \\
(\%)\end{array}$ \\
\hline Mospomorist & Normal & $65,97 \mathrm{e}$ & $13,97 \mathrm{bc}$ & $72,61 d$ & $63,6 \mathrm{de}$ & $16,49 \mathrm{c}$ \\
\hline Floradade & Normal & $92,91 \mathrm{~d}$ & $13,68 \mathrm{bc}$ & $135,36 \mathrm{a}$ & $70,1 b c$ & $25,02 \mathrm{a}$ \\
\hline $\mathrm{F}_{1}$ (Floradade $\mathrm{x}$ Mospomorist) & Normal & $129,37 \mathrm{abc}$ & $21,04 \mathrm{ab}$ & $103,63 b$ & 65,1 cde & $21,44 b$ \\
\hline $\mathrm{F}_{1}$ (TOM-596 x Mospomorist) & Heterozigoto $o g^{c}$ & $113,53 \mathrm{bcd}$ & $23,19 \mathrm{a}$ & $89,80 \mathrm{bc}$ & $63,0 \mathrm{e}$ & $21,91 \mathrm{~b}$ \\
\hline $\mathrm{F}_{1}$ (TOM-588 x Mospomorist) & Heterozigoto $0 g^{c} \& h p$ & $118,93 \mathrm{abcd}$ & $21,41 \mathrm{ab}$ & $92,82 \mathrm{bc}$ & $63,7 \mathrm{de}$ & $21,30 \mathrm{~b}$ \\
\hline $\mathrm{F}_{1}$ (TOM $559 \times$ Mospomorist) & Heterozigoto nor $^{A}$ & $107,95 \mathrm{dc}$ & $14,33 \mathrm{bc}$ & $83,97 \mathrm{~cd}$ & $70,0 \mathrm{bc}$ & $20,94 b$ \\
\hline $\mathrm{F}_{1}$ (TOM-589 x Mospomorist) & Heterozigoto $n o r^{A} \& o g^{c} \& h p$ & $139,07 \mathrm{ab}$ & $14,35 \mathrm{bc}$ & $104,17 b$ & $70,8 b$ & $21,44 b$ \\
\hline $\mathrm{F}_{1}$ (TOM-613 x Mospomorist) & Heterozigoto nor & $120,51 \mathrm{abc}$ & $18,67 \mathrm{ab}$ & $105,55 \mathrm{~b}$ & $66,7 \mathrm{bcde}$ & $20,59 b$ \\
\hline $\mathrm{F}_{1}$ (TOM-614 x Mospomorist) & Heterozigoto rin & $132,89 \mathrm{abc}$ & $16,69 \mathrm{ab}$ & $99,39 b c$ & $68,8 \mathrm{bcd}$ & $21,92 b$ \\
\hline Carmen $\mathrm{F}_{1}$ & Híbrido testemunha rin & $142,75 \mathrm{a}$ & $7,97 \mathrm{c}$ & $121,29 a$ & $77,7 \mathrm{a}$ & $17,03 \mathrm{c}$ \\
\hline Chronos $\mathrm{F}_{1}$ & Híbrido testemunha rin & $116,98 \mathrm{abcd}$ & $19,17 \mathrm{ab}$ & $122,30 \mathrm{a}$ & $69,8 \mathrm{bc}$ & $16,58 \mathrm{c}$ \\
\hline \multicolumn{2}{|l|}{ Média dos tratamentos } & 116,44 & 16,77 & 102,81 & 68,1 & 20,42 \\
\hline \multicolumn{4}{|l|}{ Contrastes de interesse } & \multicolumn{3}{|l|}{ Estimativas } \\
\hline \multicolumn{2}{|c|}{$\mathrm{F}_{1}$ vs. [(Floradade+Mospomorist)/2] } & $49,93^{* *}$ & $7,21^{*}$ & $-0,35^{\mathrm{ns}}$ & $-1,7^{\mathrm{ns}}$ & $0,68^{\text {ns }}$ \\
\hline \multicolumn{2}{|c|}{ nor $^{+} /$nor $^{A}$ vs. normal } & $-21,42^{\text {ns }}$ & $-6,71^{\mathrm{ns}}$ & $-19,6^{*}$ & $4,9^{*}$ & $-0,49^{\text {ns }}$ \\
\hline \multicolumn{2}{|l|}{ nor ${ }^{+} /$nor vs. normal } & $-8,86^{\mathrm{ns}}$ & $-2,36^{\mathrm{ns}}$ & $1,91^{\text {ns }}$ & $1,5^{\mathrm{ns}}$ & $-0,84^{\mathrm{ns}}$ \\
\hline \multicolumn{2}{|l|}{$\mathrm{rin}^{+} / \mathrm{rin}$ vs. normal } & $3,51^{\mathrm{ns}}$ & $-4,35^{\mathrm{ns}}$ & $-4,24^{\mathrm{ns}}$ & $3,6^{\text {ns }}$ & $0,48^{\text {ns }}$ \\
\hline \multicolumn{2}{|l|}{$o g^{c^{+}} / o g^{c}$ vs. normal } & $-15,84^{\mathrm{ns}}$ & $2,15^{\mathrm{ns}}$ & $-13,83^{\text {ns }}$ & $-2,1^{\mathrm{ns}}$ & $0,46^{\text {ns }}$ \\
\hline \multicolumn{2}{|l|}{$o g^{c+} / o g^{c} h p^{+} / h p$ vs. normal } & $-10,44^{\mathrm{ns}}$ & $0,36^{\mathrm{ns}}$ & $-10,80^{\text {ns }}$ & $-1,4^{\mathrm{ns}}$ & $-0,13^{\text {ns }}$ \\
\hline \multicolumn{2}{|c|}{ nor $/$ nor ${ }^{A}{ }^{\prime} g^{c+} / o g^{c} h p^{+} / h p$ vs. normal } & $9,69^{\text {ns }}$ & $-6,68^{\text {ns }}$ & $0,53^{\text {ns }}$ & $5,7^{-1,4}$ & $0,00^{\text {ns }}$ \\
\hline \multicolumn{2}{|c|}{ nor $/$ nor ${ }^{A} g^{c+} / \lg ^{c} h p^{+} / h p$ vs. nor $^{+} /$nor $^{A}$} & $31,11^{*}$ & $0,02^{\mathrm{ns}}$ & $20,19^{*}$ & $0,8^{\text {ns }}$ & $0,50^{\text {ns }}$ \\
\hline \multicolumn{2}{|c|}{ nor $/$ nor ${ }^{A} g^{c+} / o g^{c} h p^{+} / h p$ vs. $o g^{c+} / o g^{c} h p^{+} / h p$} & $20,13^{\text {ns }}$ & $-7,05^{*}$ & $11,34^{\text {ns }}$ & $7,1^{* *}$ & $0,14^{\mathrm{ns}}$ \\
\hline \multicolumn{2}{|c|}{ nor $^{+} /$nor vs. nor $^{+} /$nor $^{A}$} & $12,55^{\text {ns }}$ & $4,34^{\mathrm{ns}}$ & $21,57^{* *}$ & $-3,3^{\text {ns }}$ & $-0,34^{\text {ns }}$ \\
\hline \multicolumn{2}{|l|}{ rin $^{+} /$rin vs. nor $^{+} /$nor $^{A}$} & $24,93^{*}$ & $2,36^{\text {ns }}$ & $15,41^{*}$ & $-1,2^{\text {ns }}$ & $0.97^{\text {ns }}$ \\
\hline \multicolumn{2}{|l|}{ nor $^{+} /$nor vs. rin $^{+} /$rin } & $-12,38^{\text {ns }}$ & $1,98^{\text {ns }}$ & $6,16^{\mathrm{ns}}$ & $-2,1^{\text {ns }}$ & $-1,32^{\text {ns }}$ \\
\hline \multicolumn{2}{|c|}{ Carmen vs. $\mathrm{F}_{1}$ (Floradade x Mospomorist) } & $13,37^{\mathrm{ns}}$ & $-13,07^{* *}$ & $17,66^{*}$ & $12,6^{* *}$ & $-4,41^{* *}$ \\
\hline \multicolumn{2}{|c|}{ Carmen vs. $\mathrm{rin}^{+} / \mathrm{rin}$} & $9,86^{\mathrm{ns}}$ & $-8,72^{*}$ & $21,90^{* *}$ & $8,9^{* *}$ & $-4,89^{* *}$ \\
\hline \multicolumn{2}{|c|}{ Chronos vs. $\mathrm{F}_{1}$ (Floradade x Mospomorist) } & $-12,39^{\mathrm{ns}}$ & $-1,86^{\mathrm{ns}}$ & $18,66^{*}$ & $4,7^{\mathrm{ns}}$ & $-4,86^{* *}$ \\
\hline \multicolumn{2}{|c|}{ Chronos vs. $\mathrm{rin}^{+} / \mathrm{rin}$} & $-15,90^{\text {ns }}$ & $2,48^{\text {ns }}$ & $22,91^{* *}$ & $1,0^{\text {ns }}$ & $-5,34^{* *}$ \\
\hline
\end{tabular}

(1)Médias seguidas da mesma letra não diferem entre si a 5\% de probabilidade pelo teste de Duncan. ns Não-significativo. ${ }^{*}$ e ${ }^{* *}$ Significativo a $5 \%$ e a $1 \%$ de probabilidade, respectivamente, pelo teste F. 
O genótipo $o g^{c+} / \operatorname{og}^{c}$ e a combinação $o g^{c+} / \operatorname{og}^{c} h p^{+} / h p$ não afetaram a coloração externa dos frutos, assim como não foi observado efeito da combinação $o g^{c+} / o g^{c} h p^{+} / h p$ na coloração de frutos $n o r^{+} /$nor $^{A}$. A combinação nor ${ }^{+} /$nor $^{A}$ og $^{C^{+}} / \operatorname{og}^{c} h p^{+} / h p$ retardou a chegada da coloração vermelha nos frutos em relação ao genótipo normal.

Quanto ao aspecto produtividade, foi verificado que o background do híbrido Carmen $\mathrm{F}_{1}$, quando comparado ao background FloraDade x Mospomorist, proporcionou maior massa média por fruto (Tabela 1). A produção precoce de frutos foi afetada negativamente pelo background Carmen $\mathrm{F}_{1}$. O background do híbrido Chronos $F_{1}$ aumentou a massa média por fruto em relação ao background FloraDade x Mospomorist, não afetando as demais características de produção. Quanto ao aspecto da qualidade, os backgrounds Carmen $\mathrm{F}_{1}$ e Chronos $\mathrm{F}_{1}$ proporcionaram frutos com menor cicatriz peduncular. Foi demonstrado que, tanto o genótipo $\mathrm{rin}^{+} /$rin quanto o background genotípico favorável são responsáveis pela reconhecida alta capacidade de conservação pós-colheita dos híbridos comerciais Carmen $\mathrm{F}_{1}$ e Chronos $\mathrm{F}_{1}$.

Observa-se que a maior vida pós-colheita dos frutos dos híbridos Carmen $\mathrm{F}_{1}$ e Chronos $\mathrm{F}_{1}$ pode estar associada, em parte, ao menor tamanho relativo da cicatriz peduncular dos frutos proporcionado pelos backgrounds Carmen $\mathrm{F}_{1}$ e Chronos $\mathrm{F}_{1}$, visto que o genótipo $\mathrm{rin}^{+} /$rin não afetou significativamente o tamanho relativo da cicatriz peduncular dos frutos. Faria et al. (2003), avaliando diversos híbridos de genótipo FloraDade $\mathrm{x}$ Mospomorist juntamente com os híbridos comerciais Carmen $F_{1}$ e Chronos $F_{1}$, verificaram que estes últimos apresentaram os menores valores médios de tamanho relativo da cicatriz peduncular. Já Santos-Júnior (2002) relatou que o background do híbrido Carmen $\mathrm{F}_{1}$ proporcionou frutos com menor cicatriz peduncular e com maior longevidade em pós-colheita. Outra grande vantagem do background Carmen $\mathrm{F}_{1}$ em relação ao

Tabela 2. Valores médios relativos à firmeza inicial, meia vida da firmeza (MVF), número médio de dias para atingir firmeza 3,0x10 $\mathrm{N} \mathrm{m}^{-2}$, número médio de dias para atingir notas de coloração 4,0 e 5,0 e contrastes não-ortogonais de interesse entre 11 genótipos de tomateiro(1).

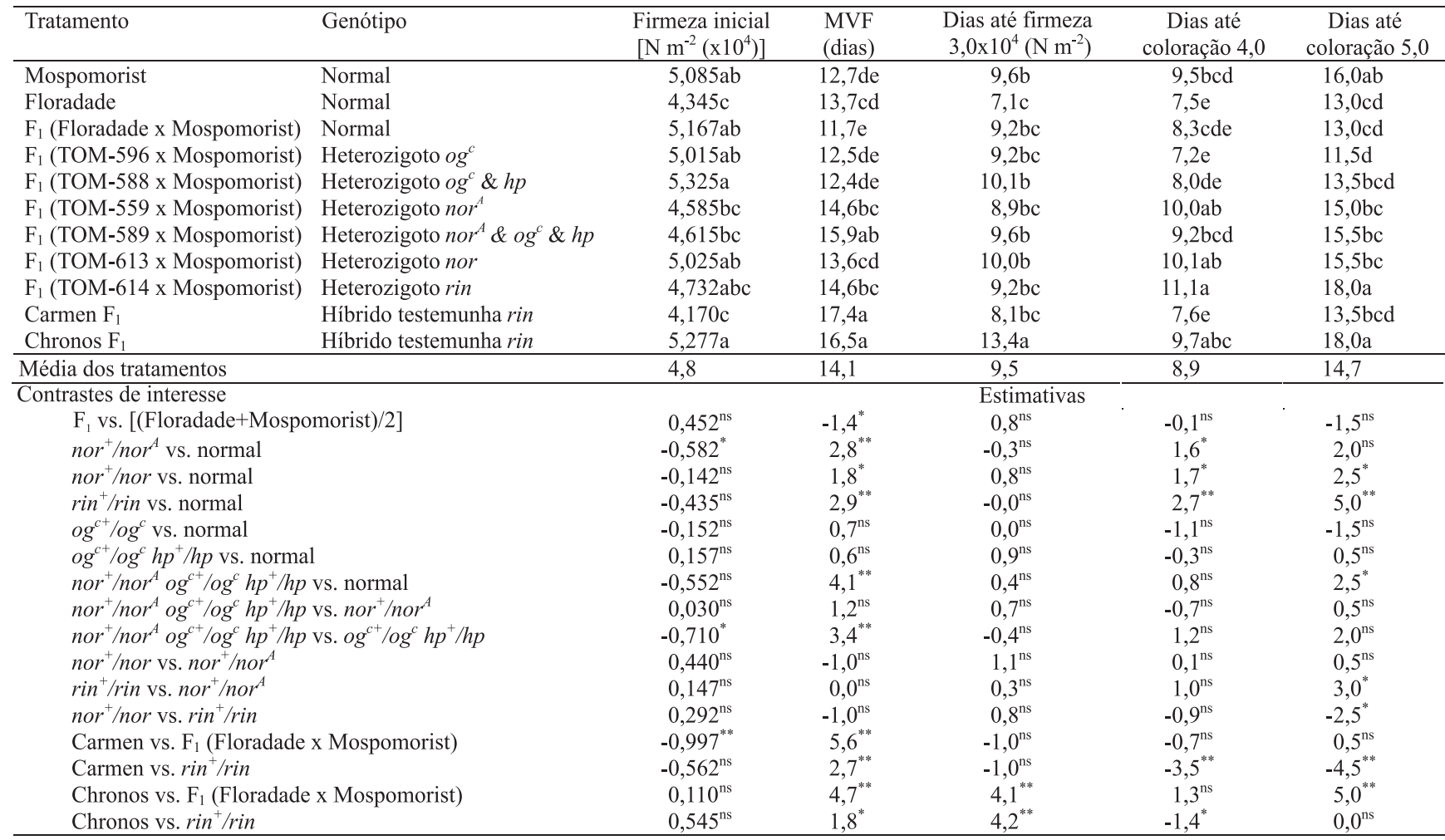

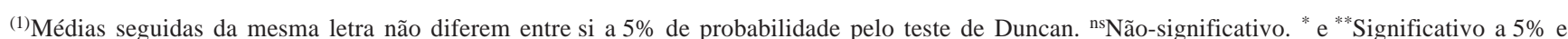
a $1 \%$ de probabilidade, respectivamente, pelo teste F. 
background FloraDade x Mospomorist é proporcionar um adiantamento na chegada da coloração vermelha dos frutos, visto que o genótipo $\mathrm{rin}^{+} / \mathrm{rin}$, isoladamente, retardou a chegada da coloração vermelha externa dos frutos. O background Chronos $\mathrm{F}_{1}$ proporcionou um ligeiro adiantamento na chegada da coloração vermelha dos frutos em relação ao background FloraDade $\mathrm{x}$ Mospomorist (Tabela 2).

\section{Conclusões}

1. Os genótipos nor $/$ nor e $\mathrm{rin}^{+} /$rin não afetam as características de produção dos híbridos avaliados; já o genótipo nor $^{+} /$nor $^{A}$ proporciona menor massa média por fruto; na combinação nor $^{+} /$nor $^{A} \mathrm{og}^{c^{+}} / \mathrm{og}^{c} \mathrm{hp} \mathrm{p}^{+} / \mathrm{hp}$, o genótipo nor $^{+} /$nor $^{A}$ não apresenta efeito negativo sobre as características de produção.

2. Os alelos nor ${ }^{A}$, nor e rin, em heterozigose, isoladamente, retardam a perda de firmeza dos frutos no background utilizado, não havendo diferenças significativas sobre esta característica; os genótipos nor ${ }^{+} /$nor $^{A}$, $\mathrm{nor}^{+} /$nor e $\mathrm{rin}^{+} /$rin retardam a chegada da coloração vermelha nos frutos, sendo o efeito do $\mathrm{rin}^{+} / \mathrm{rin}$ mais pronunciado; a coloração externa e a firmeza dos frutos não são afetadas pelo genótipo $\mathrm{og}^{c+} / \mathrm{og}^{c}$ e pela combinação $o g^{c^{+}} / \mathrm{og}^{c} h p^{+} / \mathrm{hp}$.

3. O background genotípico e a interação background $\mathrm{x}$ mutante de amadurecimento devem ser considerados na produção de híbrido $\mathrm{F}_{1}$ de tomateiro tipo longa-vida.

\section{Agradecimentos}

À Ufla, Departamentos de Agricultura e Química, pela oportunidade concedida; à Capes e à Fapemig, pelo apoio financeiro; à HortiAgro Sementes Ltda., pelo apoio na condução e realização deste trabalho.

\section{Referências}

ANDRADE-JÚNIOR, V.C.; MALUF, W.R.; AZEVEDO, S.M.; GOMES, L.A.A.; FARIA, M.V. Avaliação do potencial agronômico e da firmeza pós-colheita de frutos em híbridos de tomateiro. Ciência e Agrotecnologia, v.25, p.489-502, 2001.

ARAUJO, M.L.; MALUF, W.R.; GOMES, L.A.A.; OLIVEIRA, A.C.B. Intra and interlocus interactions between alcobaça (alc), crimson (ogc) and high pigment ( $h p$ ) loci in tomato Lycopersicon esculentum Mill. Euphytica, v.125, p.215-226, 2002.

BENITES, F.R.G. Estudos genéticos-fisiológicos dos mutantes, alcobaça (alc), non-ripening (nor) e ripening-inhibitor (rin) em tomateiro. 2003. 106p. Dissertação (Mestrado) - Universidade Federal de Lavras, Lavras.

CALBO, A.G.; NERY, A.A. Medida de firmeza em hortaliças pela técnica de aplanação. Horticultura Brasileira, v.13, p.14-18, 1995.

FARIA, M.V.; MALUF, W.R.; AZEVEDO, S.M.; ANDRADEJÚNIOR, V.C.; GOMES, L.A.A.; MORETTO, P.; LICURSI, V. Yield and post-harvest quality of tomato hybrids heterozygous at the loci alcobaça, old gold-crimson or high pigment. Genetics and Molecular Research, v.2, p.317-327, 2003.

FREITAS, J.A.; MALUF, W.R.; GOMES, L.A.A.; AZEVEDO, S.M. Efeitos dos alelos alc $\operatorname{og}^{\mathrm{c}} \mathrm{e}$ hp sobre as características de maturação e conservação pós-colheita de frutos de tomateiro. Ciência e Agrotecnologia, v.23, p.569-577, 1999.

FREITAS, J.A.; MALUF, W.R.; GOMES, L.A.A; OLIVEIRA, A.C.B.; MARTINS, W.S.; BRAGA, R.S. Padrão de amadurecimento e conservação pós-colheita de frutos de tomateiro, em função das diferentes constituições genotípicas no loco alcobaça. Revista Brasileira de Fisiologia Vegetal, v.10, p.191-196, 1998.

KOPELIOVITCH, E.; RABINOWITCH, H.D.; MIZRAHI, Y.; KEDAR, N. The potential of ripening mutants for extending the storage life of the tomato fruit. Euphytica, v.28, p.99-104, 1979.

LEAL, N.R.; MIZUBUTI, A. Características e conservação natural pós-colheita de frutos de híbridos entre a introdução 'alcobaça' e alguns cultivares de tomate. Experientiae, v.19, p.239-257, 1975.

LOBO, M. Genetic and physiological studies of the "Alcobaça” tomato ripening mutant. 1981. 107p. Thesis (Ph.D.) - University of Florida, Flórida.

MUSTSCHLER, M.A. Inheritance and linkage of the 'Alcobaça' ripening mutant in tomato. Journal of American Society for Horticultural Science, v.109, p.500-503, 1984.

RESENDE, J.M.; CHITARRA, M.I.; MALUF, W.R.; CHITARRA, A.B. Qualidade pós-colheita de genótipos de tomateiro do grupo multilocular. Horticultura Brasileira, v.15, p.92-98, 1997.

SANTOS-JÚNIOR, A.M. Produtividade, qualidade e conservação de frutos de híbridos de tomateiro heterozigotos nos locos alcobaça, non ripening e ripening inhibitor. 2002. 86p. Tese (Doutorado) - Universidade Federal de Lavras, Lavras.

Recebido em 13 de maio de 2004 e aprovado em 13 de dezembro de 2004 